Supporting Information for

\title{
Magnetic and Biocompatible Fullerenol/Fe(III) Microcapsules with Antioxidant Activities
}

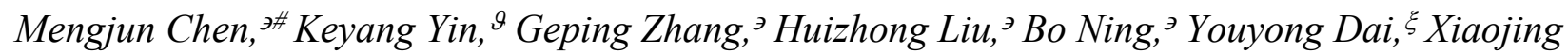
Wang, ${ }^{\dagger}$ Hongguang $\mathrm{Li}^{* \ni}$ and Jingcheng Hao ${ }^{* \ni}$

${ }^{\ominus}$ Key Laboratory of Colloid and Interface Chemistry \& Key Laboratory of Special Aggregated Materials, Shandong University, Ministry of education, Jinan, 250100, China.

\#School of Qilu Transportation, Shandong University, Jinan, 250002, China.

${ }^{\vartheta}$ State Key Laboratory of Solid Lubrication, Lanzhou Institute of Chemical Physics, Chinese Academy of Sciences, Lanzhou, 730000, China.

${ }^{\xi}$ School of Physics, Shandong University, Jinan, 250100, China.

${ }^{\dagger}$ Department of Cell Biology and Neurobiology, School of Basic Medical Sciences, Shandong University, Jinan, 250012, China

* Corresponding authors:

hgli@sdu.edu.cn Phone: 0531-88363597 Fax: 0531-88364750

jhao@sdu.edu.cn Phone: 0531-88366074 Fax: 0531-88364750 

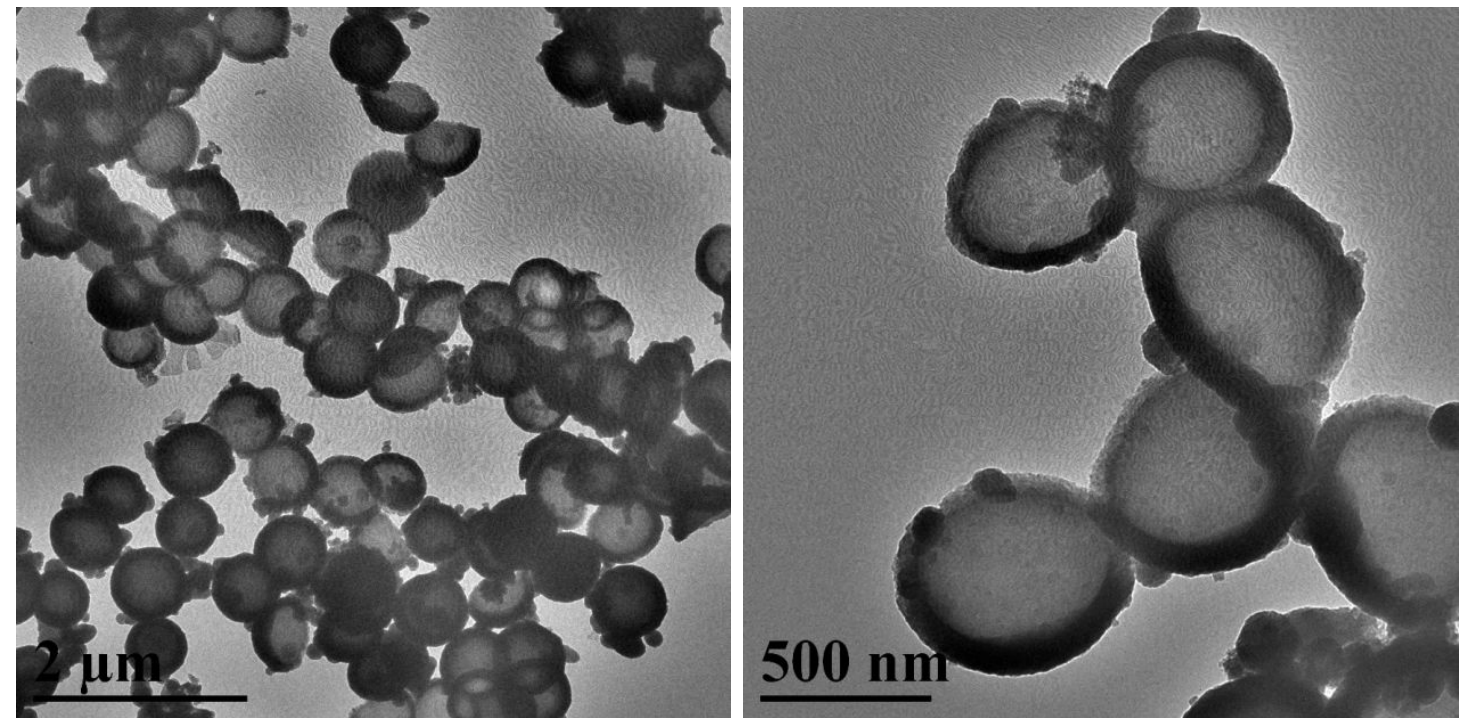

Figure S1. TEM images at different magnifications of the suspension shown in Figure la aged for $3 \mathrm{~h}$.

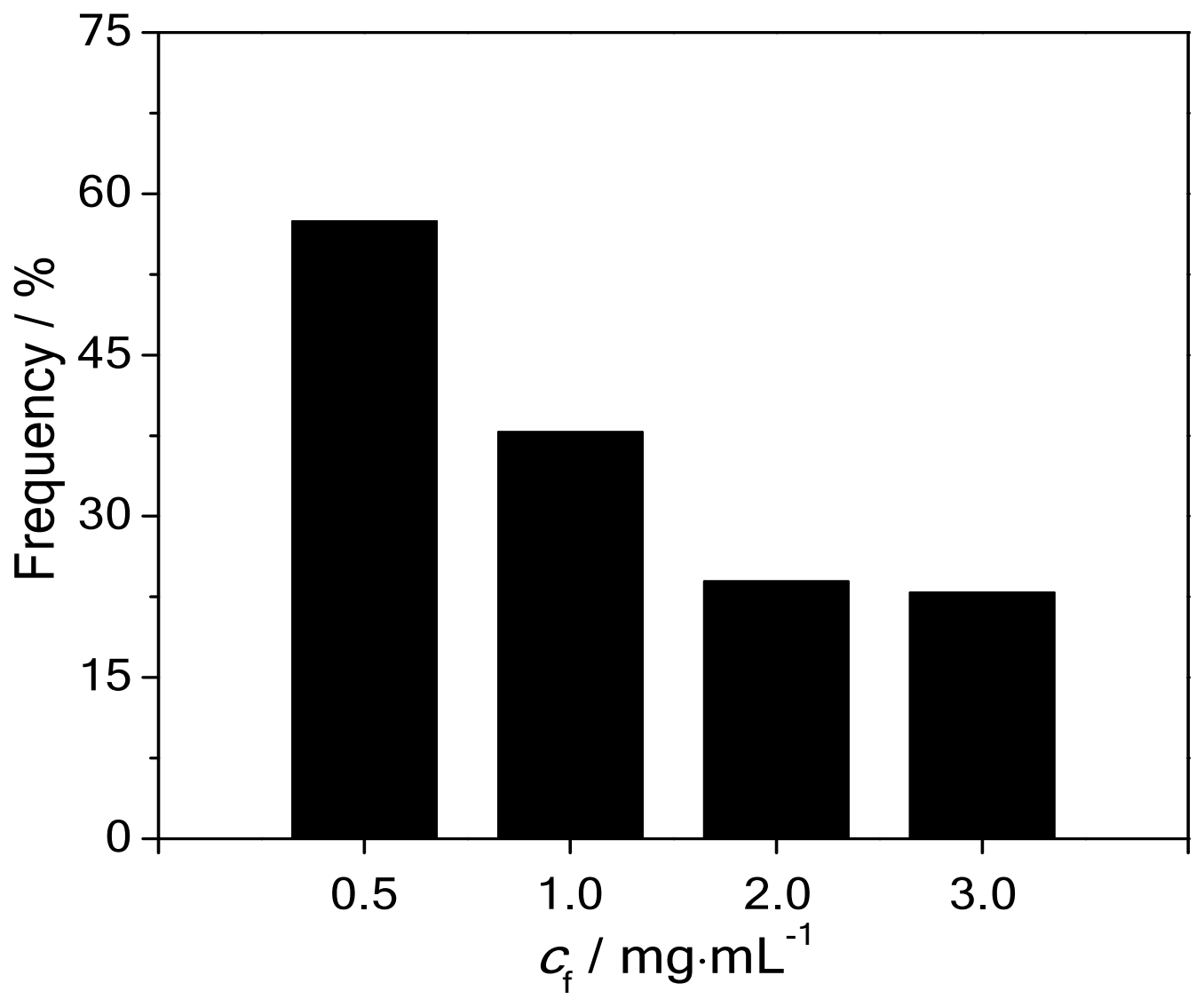

Figure S2. Statistics of the rate of cracked products for the microcapsules obtained at different $c_{\mathrm{f}}$. 


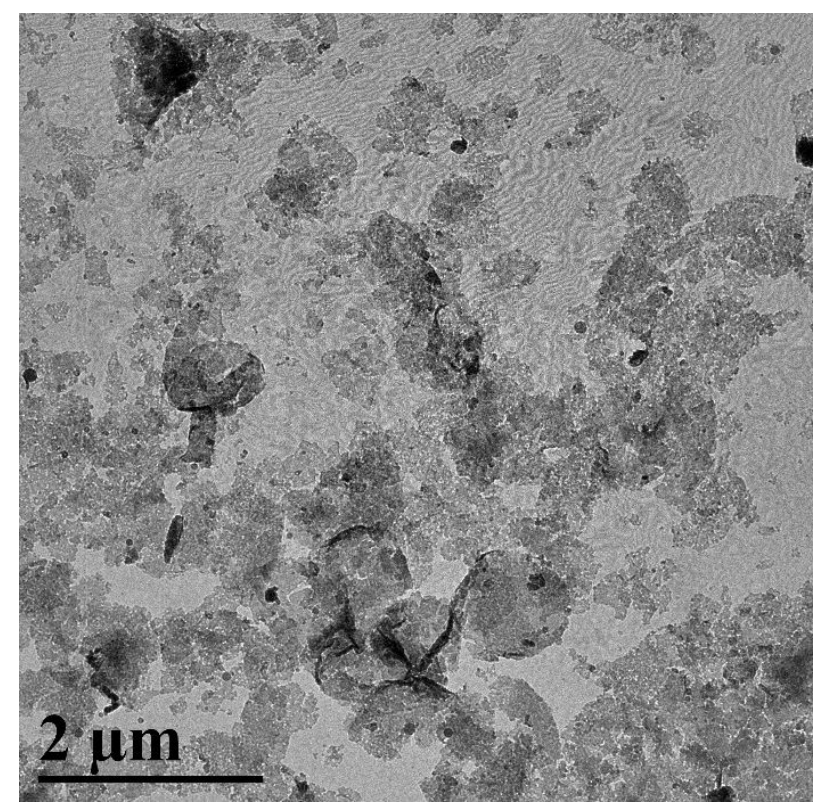

Figure S3. TEM image of the suspensions aged for $2 \mathrm{~h}$ with $c_{\mathrm{f}}=0.2 \mathrm{mg} \cdot \mathrm{mL}^{-1}$.

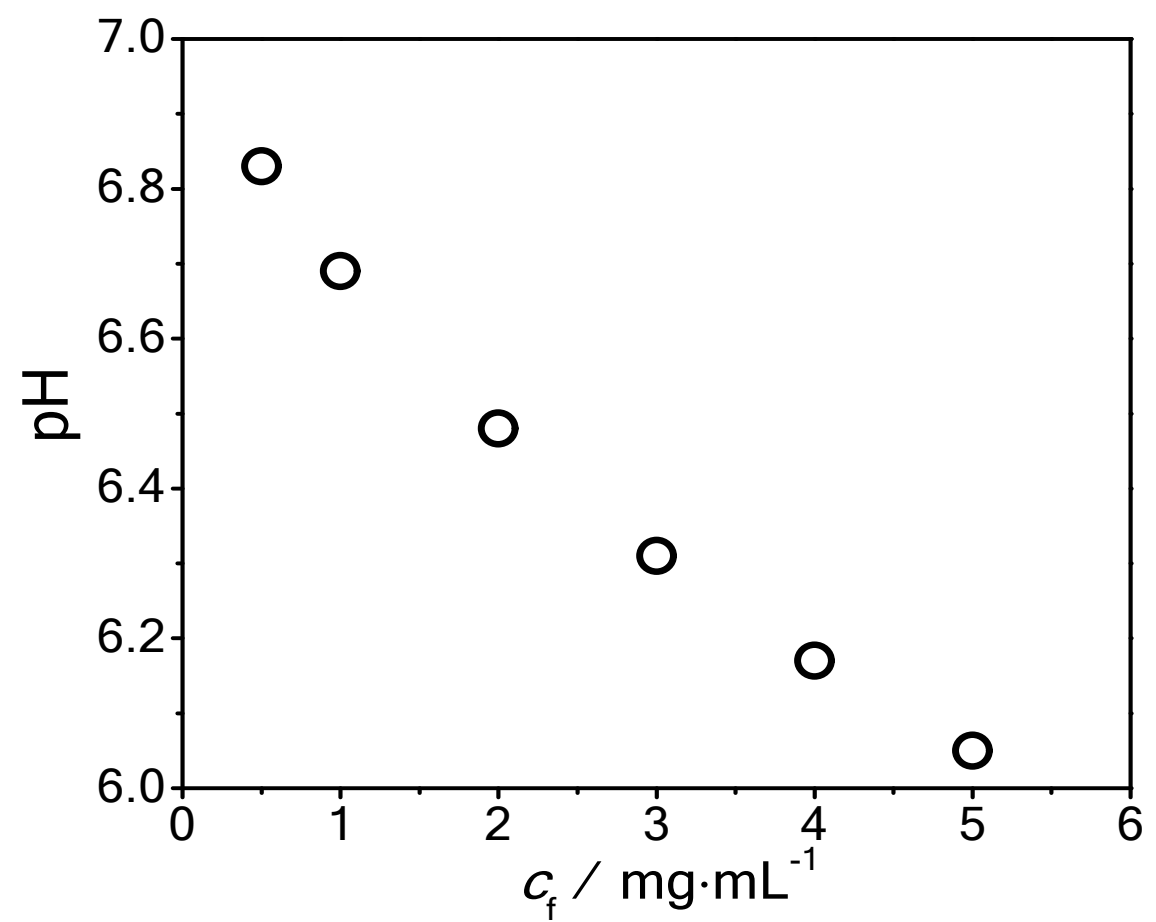

Figure S4. Variation of $\mathrm{pH}$ as a function of $c_{\mathrm{f}}$ for aqueous dispersion of the microcapsule. 

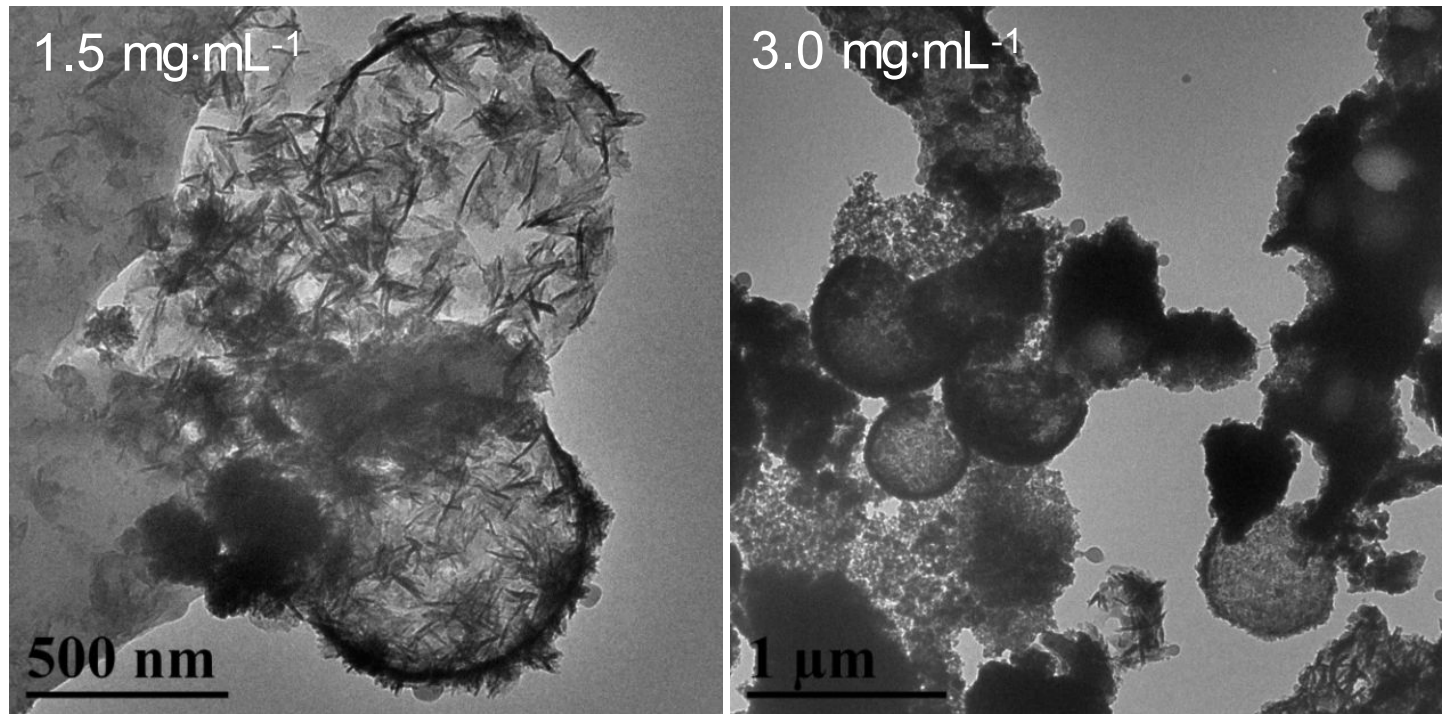

Figure S5. TEM images of the suspensions aged for $2 \mathrm{~h}$ with different $c_{\mathrm{f}}$ as indicated.

The aqueous solutions of fullerenol do not contain $\mathrm{HCl}$ and the $\mathrm{pH}$ value is 6.3-6.6.

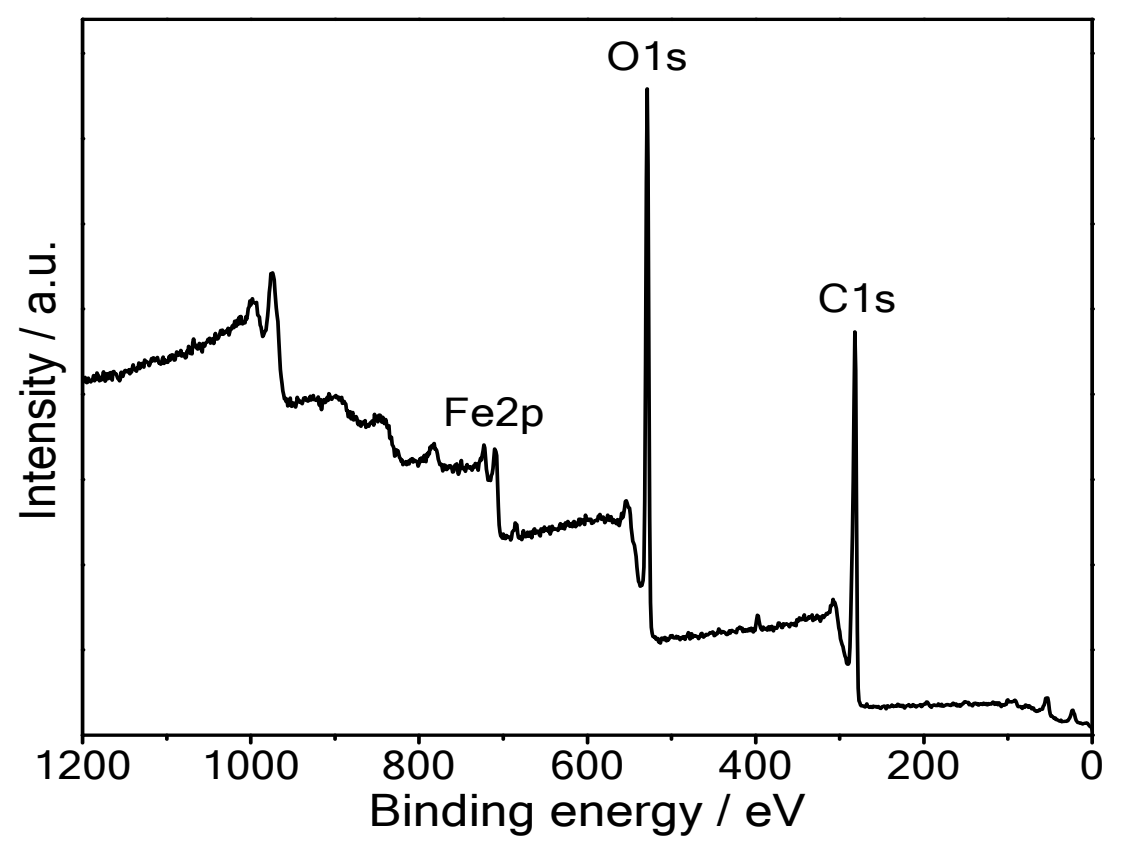

Figure S6. XPS spectrum of the microcapsule. 


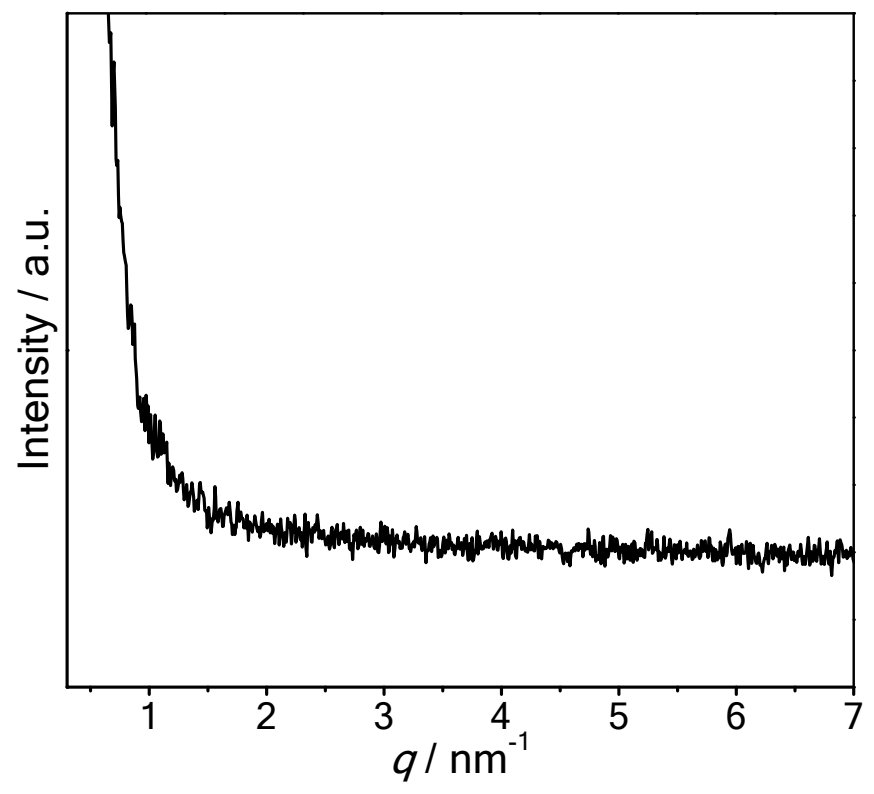

Figure S7. SAXS patten of the microcapsule.
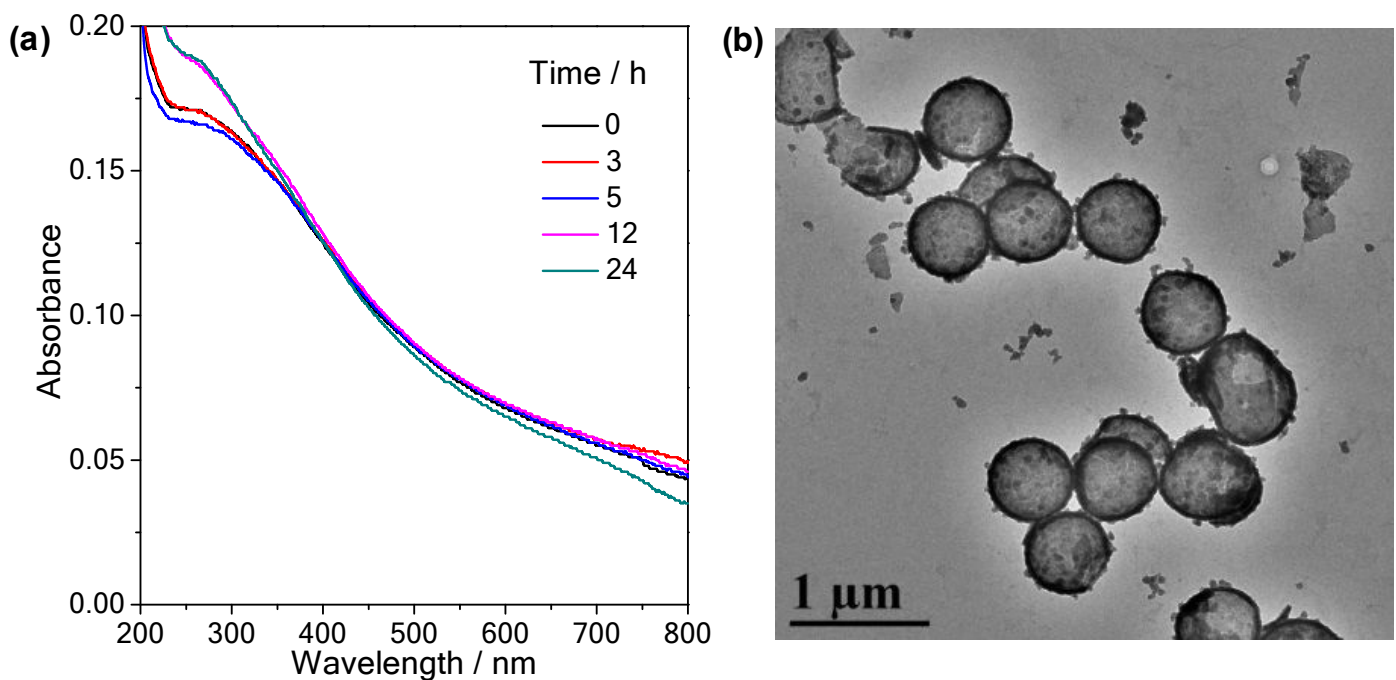

Figure S8. (a) UV-vis spectra recorded at different time for the microcapsules dispersed in aqueous solution with $\mathrm{pH}=9$. (b) TEM image of the microcapsules dispersed in aqueous solution with $\mathrm{pH}=9$. Recorded 3 days after sample preparation. 


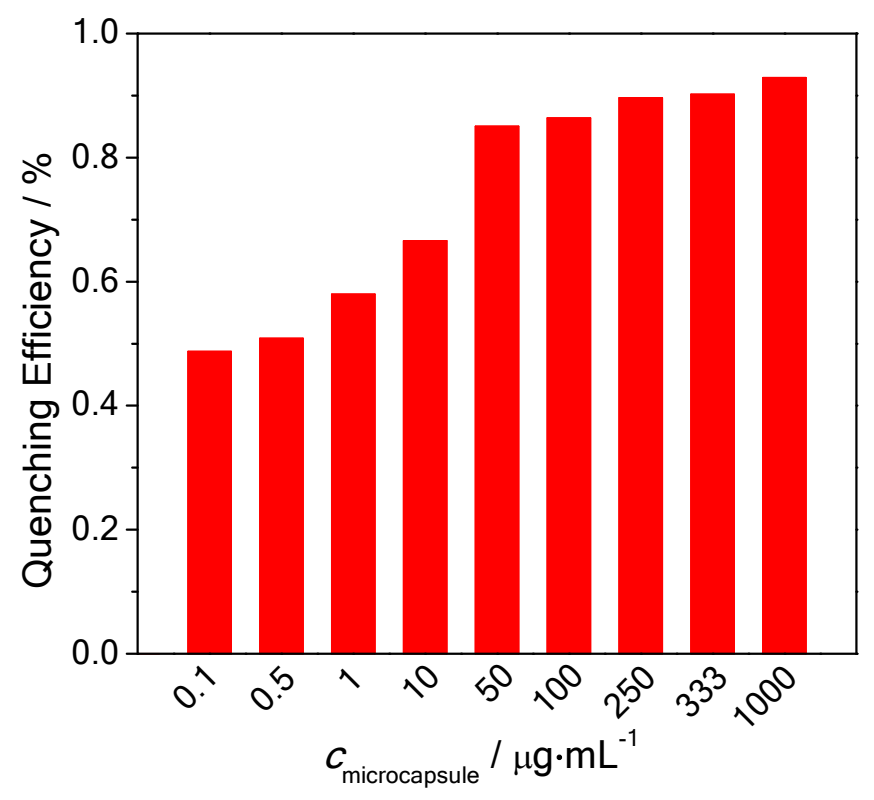

Figure S9. The quenching efficiency of $\mathrm{OH} \cdot \cdot^{-}$by the fullerenol/ $/ \mathrm{Fe}^{3+}$ microcapsule at various concentrations. 